This document is the Accepted Manuscript version of a Published Work that appeared in final form in ACS Photonics, copyright (c) American Chemical Society after peer review and technical editing by the publisher. To access the final edited and published work see http://pubs.acs.org/articlesonrequest/AORFGVA3QFPR3FKI2AJ64UX 


\title{
Switchable Photocurrent Generation in an Ultra-thin Resonant Cavity Solar Cell
}

\author{
Maximilian Götz, Maren Lengert, Norbert Osterthun, Kai Gehrke, Martin Vehse and Carsten Agert \\ DLR Institute of Networked Energy Systems, Urban and Residential Technologies, Carl-von-Ossietzky-Str. 15, 26129 \\ Oldenburg, Germany \\ Maximilian.Goetz@dlr.de \\ $\underline{\text { Maren.Lengert@dlr.de }}$ \\ Norbert.Osterthun@dlr.de \\ Kai.Gehrke@dlr.de \\ Martin.Vehse@dlr.de \\ Carsten.Agert@dlr.de
}

\begin{abstract}
Fabry-Perot type resonant nanocavities allow for broadband enhancement of light absorption in ultra-thin absorber layers. By introducing a switchable mirror, these thin film structures can be used as unique optical devices enabling interesting applications with switchable absorption. We use a thin film photovoltaic layer stack based on an amorphous germanium absorber layer and combine it with a thin $\mathrm{Mg} / \mathrm{Pd}$ mirror to create a switchable solar cell. In this work we demonstrate, how we can switch the light absorption and hence the photocurrent generation of the thin film solar cell by changing the refractive index of $\mathrm{Mg}$, due to hydrogen absorption. Our results show, how optical resonances in the absorber can be switched "on / off" by the change of optical properties of the magnesium reflector. The multi-layer system can be switched from a light absorbing and photocurrent generating state to a transparent window state with excellent color neutrality. We emphasize our study as an important step towards the realization of switchable photovoltaic windows, which paves the way for larger scale building integrated photovoltaic applications.
\end{abstract}

\section{Keywords}

Ultra-thin absorber, amorphous germanium, photovoltaic, smart window, light trapping, switchable magnesium mirror 
For more than two decades, smart windows which enable dynamic modulation of light transmission are a subject of general research interest. ${ }^{1-5}$ Electrochromic $^{6-8}, \quad$ gasochromic $^{4}$, and thermochromic $^{9-11}$, as well as liquid crystal $^{12}$ materials are integrated into glass panes, to produce window systems for smart shading. Moreover, semitransparent thin film light absorbers are developed to design photovoltaic windows. ${ }^{13}, 14$ These windows absorb a certain fraction of light to generate electric power, but still remain transparent enough for an unobstructed view. Several studies have been made on adaptive window systems with self-powering photovoltaic layers ${ }^{15-18}$, where an active layer generates photocurrent to initiate switching from the "off" to the "on" state. However, only few switchable photovoltaic window devices have been realized which are able to create more photocurrent than required to initiate the switching process. ${ }^{19-21}$ Recently, Wheeler et al. ${ }^{20}$ demonstrated that a photothermal dissociation effect can be used to switch a perovskite absorber from an opaque to a transparent state and back. A different approach was developed by Murray et al. ${ }^{19}$, using a thin amorphous silicon (aSi) absorber which was combined with a liquid crystal (LC) layer as switchable back reflector. Both concepts exhibit an on-demand photocurrent generation and can be switched between a light absorbing and a transparent state. A remaining key issue of switchable photovoltaic windows is to enhance light absorption without decreasing the transparency in the solar cell's "off" state. Therefore, light trapping, which can be switched "on" and "off" to achieve switchable absorption, has to be applied. In this paper, we outline a novel approach for the realization of switchable photovoltaic windows. We create a switchable ultra-thin resonant optical cavity by using thin gasochromic $\mathrm{Mg} / \mathrm{Pd}$ layers as switchable mirror ${ }^{22}$ behind an ultra-thin static amorphous hydrogenated germanium (a-Ge:H) absorber layer. Instead of switching the optical properties of the absorber ${ }^{23}$, the absorption enhancement of the cavity is switched by the change of optical properties of the $\mathrm{Mg} / \mathrm{Pd}$ layer system. ${ }^{24-26}$ The refractive index of $\mathrm{Mg}$ changes, when hydrogen is absorbed. ${ }^{27-30}$ The cavity in our work is based on an a-Ge:H ultra-thin solar cell ${ }^{31}$, with the main advantage to enable the integration of new absorber materials like transition metal dichalcogenides in switchable PV windows, as shown recently. ${ }^{32}$ In this study, we demonstrate a switchable optical cavity with broadband enhanced absorption in a $5 \mathrm{~nm}$ thick a-Ge:H layer, with on demand photocurrent generation. This paves the way towards switchable photovoltaic windows, as recently introduced by our research group. ${ }^{33}$ Our work demonstrates the realization of such a switchable optical cavity with photocurrent generation.

\section{Results and Discussion}

The optical and electrical properties of the switchable device are modeled and experimentally proven in the following. Local absorption of light is modeled and compared to quantum efficiency measurements to evaluate the device as switchable solar cell

\section{Device structure and cavity effect}

The device layer stack consists of an electrical front contact, a p-i-n structure for charge carrier separation and the switchable rear contact. The device is shown in Figure 1 (a). The front contact material is aluminum doped zinc oxide (AZO). N-doped a-Si:H as well as an intrinsic a-Si buffer layer is deposited on top of it. Afterwards the a-Ge:H layer for light absorption, an intrinsic $\mu \mathrm{c}-\mathrm{Si}: \mathrm{H}$ and a p-doped $\mu \mathrm{c}$ $\mathrm{Si}: \mathrm{H}$ layer are deposited. This is covered with a thin buffer layer of $\mathrm{MoO}_{\mathrm{x}}$. The last layers are the switchable rear contact, consisting of $24 \pm 2 \mathrm{~nm} \mathrm{Mg}$ with a $6 \pm 2 \mathrm{~nm} \mathrm{Pd}$ capping layer to prevent oxidation of $\mathrm{Mg}$ and to catalyze the absorption of hydrogen. ${ }^{34,35}$ The $\mathrm{MoO}_{\mathrm{x}}$ layer is used to improve the band alignment between the low work function material $\mathrm{Mg}^{36,37}$ and $\mathrm{p}$-doped $\mu \mathrm{c}$-Si. The device is illuminated from the glass side, meaning the cell is in superstrate configuration. The optical cavity is established between $\mathrm{Mg}$ and $\mathrm{AZO}$ as will be shown in the next section. The photocurrent is also extracted from these layers. Figure 1 (b) shows a scanning electron microscopy (SEM) image of a cross-section of the layer stack. The p-i-n layers can be clearly seen in in dark grey, while the front and the rear materials appear brighter. An enlarged view of the layer stack is shown in Figure 1 (c). It is evident, that the absorber forms a closed layer on the substrate. The roughness of the layer system originates from the structure of AZO. The crystalline material introduces hills and valleys into the device. The absorber and rear contact 
follow the roughness of the front contact without disruption of the layers.

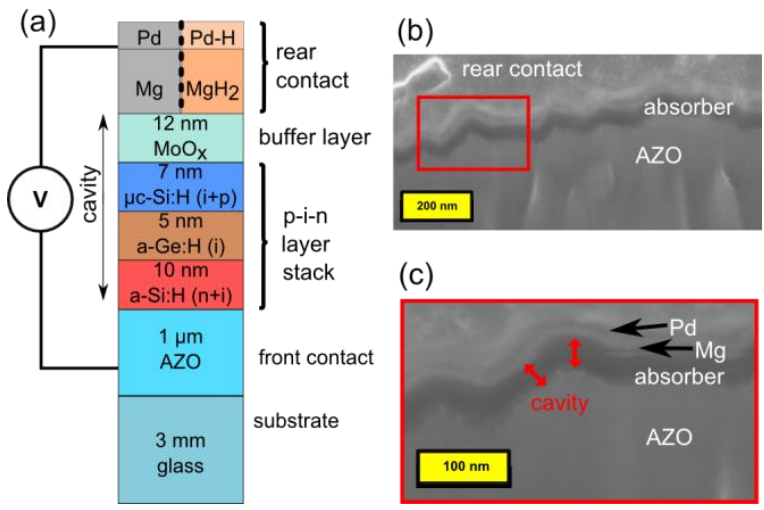

Figure 1: (a) The investigated layer stack. (b) scanning electron microscopy (SEM) images of a cross-section of the layer stack milled with focused ion beam (FIB). (c) higher magnification SEM image of cavity.

\section{Optical Properties of the Switchable Cavity}

Before showing the details of the electro-optical analysis of the device layer stack, we want to demonstrate the optical properties of the switchable cavity with a less complicated layer stack. Figure 2 (a) shows the measured transmission and reflection spectra of $30 \mathrm{~nm} \mathrm{Mg} / 5 \mathrm{~nm} \mathrm{Pd} /$ glass, together with the simulated spectra from the modeled dielectric function The refractive index data, which was used to model the spectra, is shown in the supporting information. Figure 2 (b) presents the results of the same layer stack after hydrogen uptake. The large change in reflectivity from values above $80 \%$ before hydrogen exposure to values below $10 \%$ after hydrogen exposure demonstrates the drastic change of the optical properties, which clearly indicate a transformation of the refractive index. This induced change in the refractive index will later allow for switching of the absorption in the cavity. Figure 2 (a) and (b) also present the modeled $R$ and $T$ plots for $\mathrm{Mg} / \mathrm{Pd}$ on a glass substrate. To match the measured spectra in the hydrogenated state our model assumes an absorptive mix layer on top. It includes a $\mathrm{Mg}-\mathrm{Pd}$ intermetallic phase as well as partially hydrogenated material. This phase may originate from the interdiffusion of $\mathrm{Mg}$ and $\mathrm{Pd}$ atoms during hydrogenation. ${ }^{38}$ This layer in the model accounts for the parasitic absorption of the hydrogenated layers. From simulation and measurement it becomes clear, that the parasitic absorption $1-R-T$ of the layers increases when hydrogen is incorporated in the $\mathrm{Mg} / \mathrm{Pd}$ layers. Switchable absorption in a thin absorber layer can be achieved by adding a thin (22 $\mathrm{nm}) \mathrm{a}-\mathrm{Ge}: \mathrm{H}$ layer on top of the switchable $\mathrm{Mg} / \mathrm{Pd}$ mirror. This is the simplest form of the cavity. Figure 2 (c) shows the simulated absorption spectra of this configuration. The total absorption is compared with the local absorption in the a-Ge:H layer for both states. Absorption above $60 \%$ of incoming light can be reached locally in the a-Ge:H layer with metallic $\mathrm{Mg}$ rear contact. This will be referred to as the cavity "on" state. Light is confined inside the ultra-thin absorber material due to nontrivial interferences. ${ }^{31,39-}$ ${ }^{42}$ The internal electric field distribution of the cavity depends on the phase shift at the interfaces, and the phase shift caused by the thickness of the layers. ${ }^{23}$ Broadband interferences emerge in the absorber layer on $\mathrm{Mg}$ and enhance the overall absorption of the layer stack. It can be seen that the cavity effect is drastically reduced when $\mathrm{Mg}$ is changed to $\mathrm{MgH}_{2}$. When the reflectivity of $\mathrm{Mg}$ is decreased and the transparency increases due to hydrogen absorption, the cavity effect is reduced and less light is confined in the absorber material. This can be seen in the drop of local and total absorption in Figure 2 (c). With $\mathrm{MgH}_{2}$ rear contact the local absorption in the a-Ge:H layer is below $40 \%$, which we will refer to as the cavity "off" state. This shows that our device is able to realize switchable absorption with the $\mathrm{Mg} / \mathrm{Pd}$ mirror. After hydrogen exposure, the local absorption decreases significantly compared to the total absorption at wavelengths above $800 \mathrm{~nm}$. This can be explained by the parasitic absorption of the hydrogenated layers, as discussed above. The main difference between this simplified configuration and the electrical functioning device is the thick $(1 \mu \mathrm{m})$ AZO front contact, which covers the absorber. Figure 2 (d) shows the simulated absorption spectra of this configuration on a glass substrate. It can be seen that the absorption changes between cavity "on" and "off" state. The cavity effect is still evident and the absorption can be switched. The additional interference peaks in the absorption spectra may be attributed to optical resonances within the AZO layer. In this simulation the roughness of the AZO layer, which was also seen in the SEM images was not included. The additional roughness does not decrease the cavity effect since it is angular stable ${ }^{43}$, and may 
even improve the absorption. ${ }^{44}$ After showing that switchable light absorption is achieved in a simplified layer stack, the optical properties of the device layer stack presented in Figure 1 (a) are studied. We demonstrate that the transmission and absorption of the p-i-n solar cell structure is switchable based on the same principle as shown before and validate our optical model by comparing it to experimental measurements. Figure 2 (e) shows the absorption spectra of the device layer stack in the "on" and "off" state of the cavity calculated as 1-R-T. It can be seen that the spectra are again dominated by the interference pattern of the AZO layer as already shown in Figure 2 (d). In the visible spectral region (350 to $800 \mathrm{~nm}$ ) the absorption in cavity "on" state reaches values above $70 \%$. Above $\lambda=800 \mathrm{~nm}$ the absorbance of the device in "on" state decreases. Less light is confined in the cavity, which is a result of the decreased absorption of a-Ge: $\mathrm{H}$ in this spectral region. This confirms the results from Figure 2 (c). After hydrogenation, the absorption changes significantly (blue line). The dielectric properties of $\mathrm{MgH}_{2}$ shift the modes of the cavity out of the absorber layer. The absorption drops below $70 \%$ for most of the visible spectral region. At wavelengths above $\lambda=800 \mathrm{~nm}$, the absorption of the device with dielectric rear mirror exceeds the absorption of the
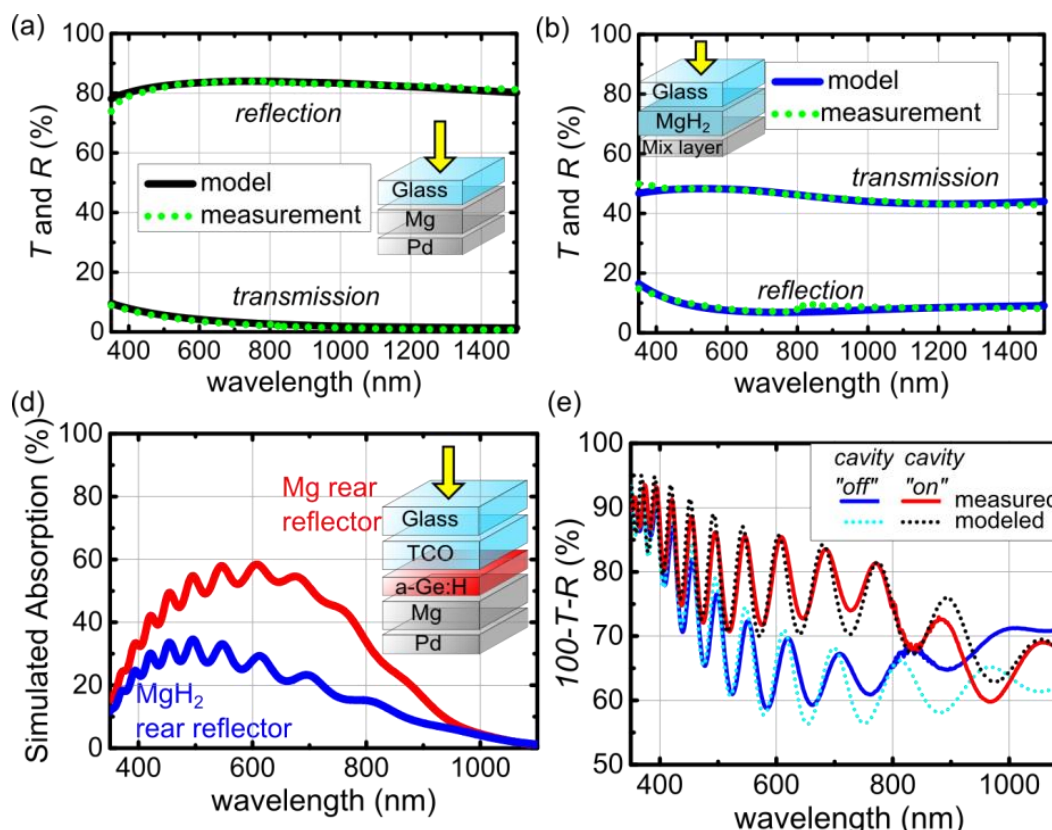

(e)

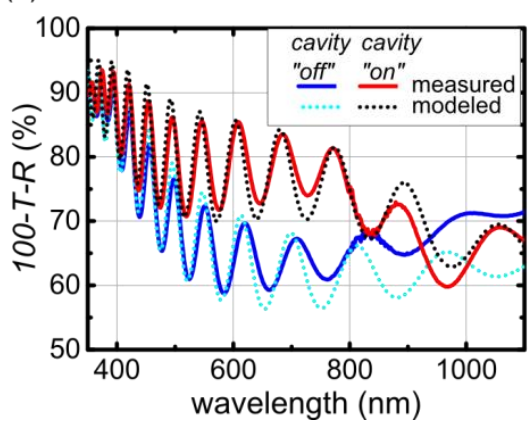

device in "on" state. A significant part of the light is absorbed in the layer mix of $\mathrm{Mg}$ and $\mathrm{Pd}$ and not confined to the cavity any more, as will be shown in Figure 3. The overall high absorption for wavelengths below $400 \mathrm{~nm}$ in both states is due to parasitic absorption in the AZO layer. The optical model (solid lines) is fitted to the measurements by adjusting the layer thickness of the respective layers. The layer thickness of $\mathrm{Mg}$ is changed in the simulation from $24 \mathrm{~nm}$ to $29 \mathrm{~nm}$ after hydrogen absorption which is an increase in thickness of $21 \%$. Previous published studies from different research groups have shown that a volume expansion of $\mathrm{Mg}$ occurs when hydrogen is absorbed. ${ }^{45-47}$ This applies also to our results. The models are in good accordance with the measurements, especially for wavelengths below $800 \mathrm{~nm}$. A reason for deviations of the model from the measurements may be inaccuracies in the modeled refractive indices, as well as differences between modeled and actual thickness. The results demonstrate that the absorption of the device is switchable. Figure 2 (f) and (g) present photographs of the cell stack in "on" and off "state". The transparency increases significantly after hydrogen absorption. The device in "on" state is only slightly transparent, showing that not all incoming light is confined to the cavity.

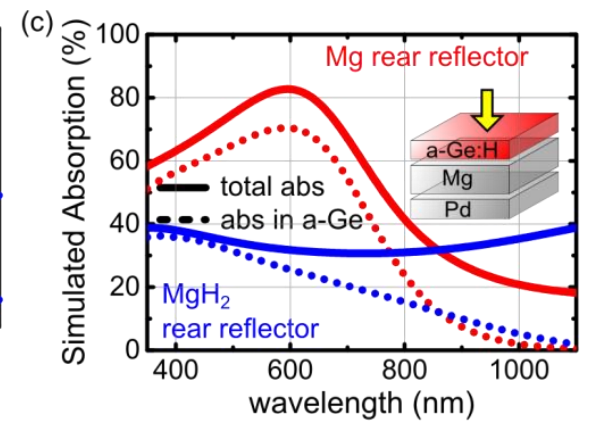

Figure 2: (a) Measured (dotted) transmittance (T) and reflectance (R) of $30 \mathrm{~nm} \mathrm{Mg}$ and $5 \mathrm{~nm}$ Pd. The solid lines present the optical model, fitted to the measurement. (b) $T$ and $R$ after exposure to 5 mbar hydrogen in nitrogen for 15 minutes. (c) Simulated absorption for a layer stack of a-Ge:H / Mg / Pd (red), as well as a-Ge:H/MgH / mix 
layer (blue) in vacuum. The total absorption of the complete layer stack is plotted with solid lines, while dotted lines shows only the absorption in the germanium layer. (d) Local absorption in Ge with glass and AZO as realistic front contact/mirror. (e) measured (solid) and modeled (dotted) absorption spectra (1-R-T) of the solar cell layer stack in "on" and "off" state. Photograph of the layer stack in "on" $f(f)$ and "off" $(g)$ state.

\section{Switchable local absorption in an ultra-thin solar cell}

After validating our model in terms of predicting the absorption and transmission measurement, we studied the local absorption in the device, using the same optical model as shown in Figure 2 (e). Figure 3 (a) and (b) show the simulated local differential absorption $\mathrm{d} A$ of each layer, starting with $\mathrm{AZO}$, the $\mathrm{Si}$ and Ge layers followed by the $\mathrm{MoO}_{\mathrm{x}}$ buffer layer and the switchable back contact. It has to be noted, that for improved readability only the lower 10 to $20 \mathrm{~nm}$ of the AZO layer are shown in these graphs. Figure 3 (a) presents the device with metallic rear reflector $\mathrm{Mg}$ / Pd. In this case, the a-Si:H, a-Ge:H and $\mu \mathrm{c}-\mathrm{Si}: \mathrm{H}$ layers show significant absorption. However, most light is absorbed in the a-Ge:H layer, which is the desired case for our solar cell application. Especially in the wavelength range between $\lambda=400$ and $800 \mathrm{~nm}$ the a-Ge:H layer dominates the absorption of light. The oscillations in the absorption originate from the interferences in the AZO. Furthermore, Figure 3 (a) provides another interesting insight into the system. Absorption is almost absent in the metallic layers $\mathrm{Mg}$ and $\mathrm{Pd}$, as expected. The absorption in $\mathrm{Mg}$ increases at wavelengths above $\lambda \approx 700 \mathrm{~nm}$. This effect is consistent with our optical measurements in Figure 2.

Figure 3 (b) shows that with the hydrogenated $\mathrm{MgH}_{2}$ mirror, less light is confined inside the cavity. It is interesting to see, that a small amount of light in the wavelength range between 350 and $450 \mathrm{~nm}$ is absorbed at the interface between TCO and a-Si:H. This can be explained by a shift of a cavity mode to this position. In the cavity "off" state, most light is absorbed in the a-Ge:H layer compared to the $\mathrm{Si}$ layers. The reason for that is the much higher absorption coefficient of a-Ge:H compared to a-Si:H and $\mu \mathrm{c}-\mathrm{Si}: \mathrm{H}$. Concerning the mirror, almost no absorption is present in $\mathrm{MgH}_{2}$, though a significant part of light is absorbed in the mix layer. This indicates that, to achieve high transparency the absorption in the mix layer has to be reduced. When comparing the absorption plots in both states, it becomes clear, that the cavity is switchable and the resonant modes are at the desired position inside the device. For simplification the layer roughness and thickness variations were not considered in our model. This means that the cavity effect varies slightly locally. Nevertheless, the simulations demonstrate how the cavity effect and with it the absorption enhancement can be switched "on" and "off".
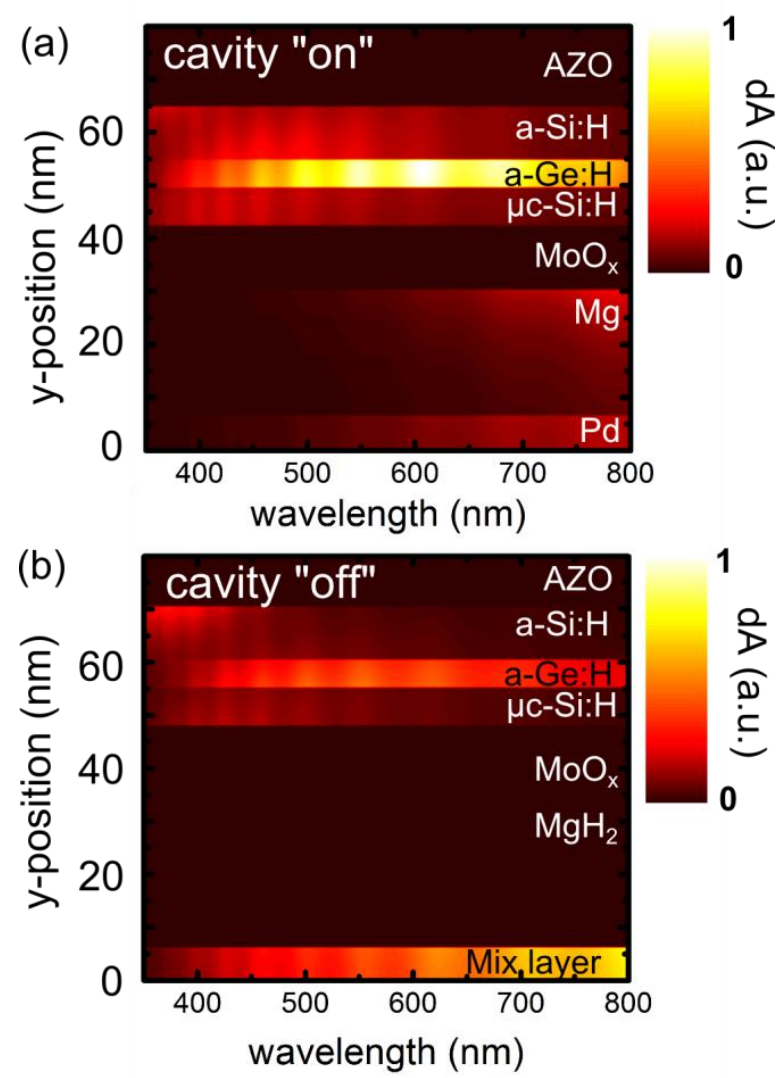

Figure 3: Color maps of the local absorption within the device layer stack with metallic rear mirror (a) and with dielectric rear mirror (b). The position inside the layer stack is shown on the y-axis, while the $x$-axis presents the wavelengths. The absorption is encoded in the color bar. 


\section{Switchable photocurrent generation}

The previous section has presented the optical properties of the device layer stack. In the following we want to show the electrical properties and present in detail how the switching of the cavity influences the current generation. Therefore, the measured external quantum efficiency (EQE) for the two states ("on/off") are compared to the simulated internal quantum efficiency (IQE). Figure 4 (a) presents the EQE and IQE spectra of the switchable device with the cavity switched "on". The EQE spectrum reaches a peak value of $40 \%$ at a wavelength $\lambda=450 \mathrm{~nm}$, meaning that photocurrent is extracted at this wavelength with highest efficiency. Simulated IQE results are plotted for a-Ge:H, $\mu \mathrm{c}-\mathrm{Si}: \mathrm{H}$ and a-Si:H layers, respectively. The a-Si and $\mu \mathrm{c}-\mathrm{Si}$ layers show an IQE of less than $15 \%$, while the IQE of the a-Ge:H layer reaches $30 \%$ at $\lambda=600 \mathrm{~nm}$. The a-Ge:H layer contributes significantly more to the photocurrent than the Si layers. Comparing the three single layer IQE spectra to the EQE, it is evident that the charge carriers from the a-Ge:H layer alone are not enough to explain the measured behavior of the EQE spectrum. Only the sum of all three IQE spectra (plotted in light blue) is able to reproduce the measured spectrum at least at low wavelengths up to $\lambda \sim 450 \mathrm{~nm}$. For $\lambda>450 \mathrm{~nm}$ the sum of the single layer IQE on the other hand is much larger than the EQE, pointing out strong recombination or optical loss in that wavelength range.

A similar effect can be seen in Figure 5 (b), where the IQE and EQE for the "cavity off" mode are shown. The comparison of the EQE measurements in both states shows, that the photocurrent of the nano cavity can be reduced by switching the rear contact from a metallic to a dielectric state, as confirmed by simulation. The IQE of the a-Ge:H layer shows a strong reduction compared to Figure 5 (a). It is interesting to see that the measured $\mathrm{EQE}$ of the "cavity off" cell exceeds the IQE at wavelengths smaller than $500 \mathrm{~nm}$. This suggests that the cavity effect may be better than predicted by simulation for small wavelengths. This can be a result of the roughness of the AZO layer, which is not implemented in the model or a rectifying contact between $\mathrm{Mg}$ and the $\mathrm{p}$-side of the solar cell.

As mentioned before, recombination and optical losses may be responsible for the low EQE at higher wavelengths. Two additional samples with alternative back contacts are prepared to study these hypotheses. To see if the thin $\mathrm{Mg} / \mathrm{Pd}$ layer is responsible for the optical loss, the switchable mirror layer is replaced by $300 \mathrm{~nm}$ of $\mathrm{Mg}$. This layer is completely opaque and no light is transmitted through the layer stack. The results of IQE simulation and EQE measurement are presented in Figure 4 (c). Similar differences between EQE and IQE as in Figure 4 (a) can be seen. The EQE is reduced compared to the IQE for wavelengths above $500 \mathrm{~nm}$ and increased for wavelengths below. The EQE of the device with $300 \mathrm{~nm}$ rear mirror is slightly improved, showing that optical losses play a role in the device with thin $\mathrm{Mg} / \mathrm{Pd}$. To see if $\mathrm{Mg}$ metal itself causes increased recombination at the interface to $\mathrm{MoO}_{\mathrm{x}}$ a device with $300 \mathrm{~nm} \mathrm{Ag}$ rear reflector is studied as well. Ag possess a much higher work function compared to $\mathrm{Mg}^{36}$, changing the band alignment of the cell stack. The results are shown in Figure 4 (d). In this case, the measured EQE fits the simulated IQE much better, with only small deviations for $\lambda>550 \mathrm{~nm}$. This confirms that optical losses, as well as recombination both contribute to the decreased EQE for wavelengths above $500 \mathrm{~nm}$. Recombination at the interface between $\mathrm{Mg}$ and $\mathrm{MoO}_{\mathrm{x}}$ and the electron selectivity of $\mathrm{Mg}^{48}$ reduce the amount of charge carriers extracted from the device. The overall higher quantum efficiency in Figure 4 shows the potential of the switchable cells to reach quantum efficiencies above $50 \%$. 

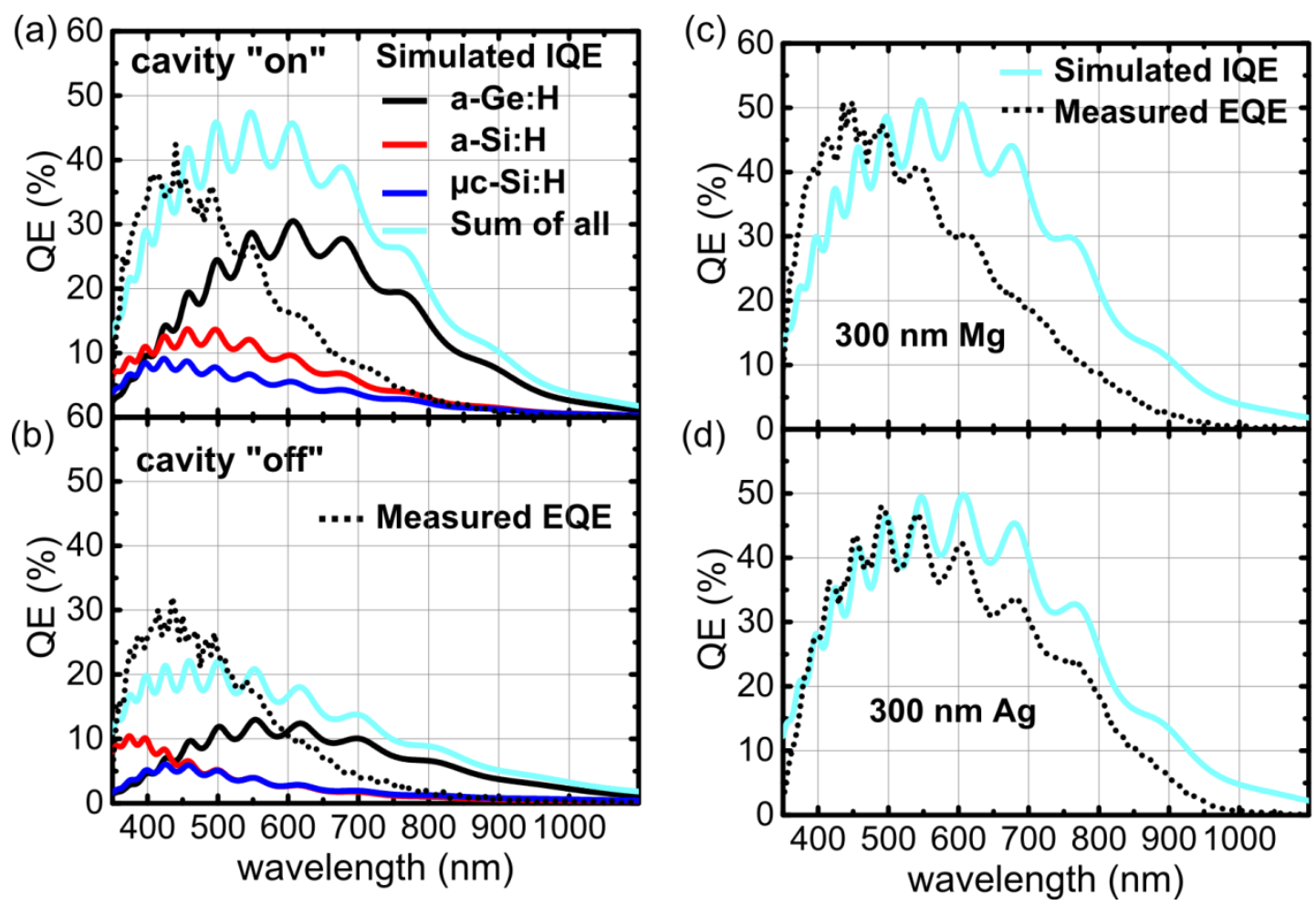

Figure 4: measured EQE and simulated IQE of the switchable device in "cavity on" (a) and "cavity off" (b) mode. Measured EQE and simulated IQE of the devices with $300 \mathrm{~nm} \mathrm{Mg} \mathrm{(c)} \mathrm{and} 300 \mathrm{~nm} \mathrm{Ag} \mathrm{rear} \mathrm{mirror} \mathrm{(d)} \mathrm{replacing} \mathrm{the}$ thin film $\mathrm{Mg} / \mathrm{Pd}$ layers.
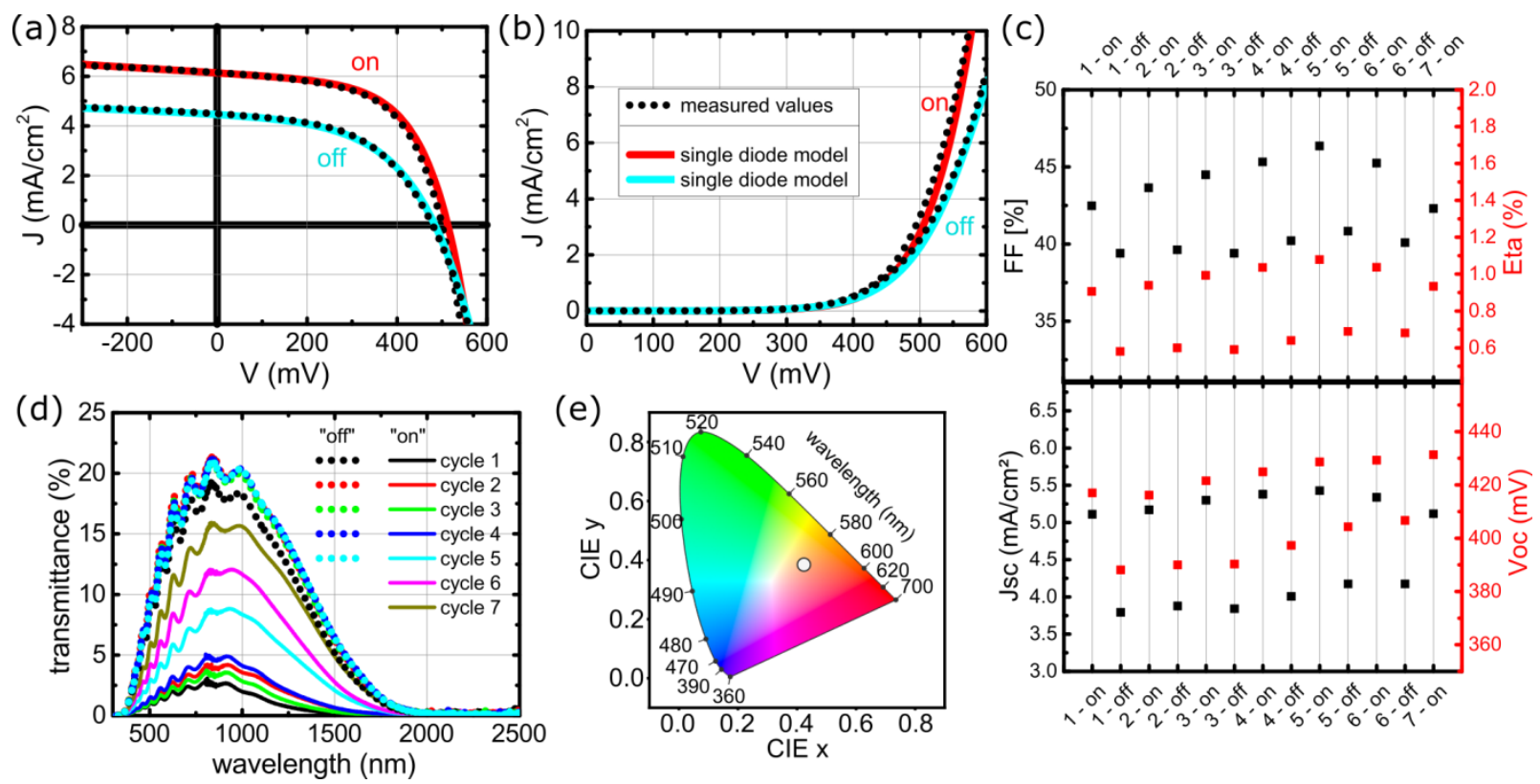

Figure 5: measured illuminated (a) and dark (b) IV curve of cell in "on" and "off" state with single diode fit. Fill factor $(F F)$, efficiency (Eta) short circuit current (Jsc) and open circuit voltage (Voc) for seven cycles of switching (c). Measured transmission at for seven cycles of switching (d).Chromaticity plot for color appearance of cell in transparent mode $(e)$. 


\section{Switchable PV window}

In order to study the PV performance of the solar cell, the IV curves of the device were measured in "on" and "off" state. The cell stack was slightly modified with a $2 \mathrm{~nm}$ thick layer of titanium above and below the $\mathrm{Mg}$ switchable layer, respectively. The Ti layer between $\mathrm{Mg}$ and $\mathrm{Pd}$ is known to reduce the deleterious effects of the alloying of $\mathrm{Mg}$ and $\mathrm{Pd}$ on the switching properties of the cell stack. ${ }^{49}$ Moreover, the $\mathrm{Ti}$ layer might minimize clamping to the underlying $\mathrm{MoO}_{\mathrm{x}}$ layer. ${ }^{47}$ This might reduce the mechanical stress due to volume expansion of $\mathrm{Mg}$ after hydrogen absorption on the $\mathrm{p}$-i-n structure. ${ }^{50}$ Figure 5 (a) presents the measured current density $(J)$ as a function of voltage $(V)$ for both states of the solar cell under AM1.5G illumination. The characteristic parameters are extracted by fitting of the single diode equation to both graphs. In the "on" state the cell reaches a short circuit current $\mathrm{J}_{\mathrm{sc}}=6.13 \mathrm{~mA} / \mathrm{cm}^{2}$ and an open circuit voltage $\mathrm{V}_{\text {oc }}=500 \mathrm{mV}$. After 20 minutes of hydrogen absorption the $\mathrm{J}_{\mathrm{sc}}$ is decreased to $\mathrm{J}_{\mathrm{sc}}=4.50 \mathrm{~mA} / \mathrm{cm}^{2}$, while $\mathrm{V}_{\mathrm{oc}}=481 \mathrm{mV}$ only shows a small reduction. The fill factor reaches $\mathrm{FF}$ ("on") $=57.6 \%$ and $\mathrm{FF}$ ("off") $=50.8 \%$, which leads to an overall efficiency of $\eta$ ("on") $=1.78 \%$ and $\eta$ ("off") $=1.01 \%$. The most significant reduction of characteristic values can be found for $\mathrm{FF}$ and $\mathrm{J}_{\mathrm{sc}}$. The reduction of $\mathrm{J}_{\mathrm{sc}}$ can be explained by the reduced absorption of light in the Ge layer. Dark IV curves were measured to study the influence of hydrogen absorption on the reduction of FF. Figure 5 (b) presents the dark IV curves of the cell. By fitting the single diode equation to these measurements, values for the series resistance $R_{s}$ can be extracted. The results are $\mathrm{R}_{\mathrm{s}}$ ("on") $=2.0 \Omega$ and $\mathrm{R}_{\mathrm{s}}$ ("off") $=5.5 \Omega$. This is also comparable to the sheet resitance of the TiMg-Ti-Pd layer stack on glass determined by four point probe measurements. Here the sample reaches a sheet resistance before exposure to hydrogen of $\mathrm{R}_{\square}$ (“"on”) $=2.7 \pm 0.1 \Omega / \square \quad$ and $\quad$ of $R_{\square}$ ("off") $=9.7 \pm 0.5 \Omega / \square$ after hydrogen absorption. The increase of the resistance after hydrogen absorption leads to the reduction of FF and to the small decrease of Voc. The sheet resistance increases, because the dielectric $\mathrm{MgH} 2$ has a smaller conductivity than pure $\mathrm{Mg}$. The decrease of $\mathrm{V}_{\mathrm{OC}}$ might also result from the lower intensity of light confined in the cell in the "off" state.

The degradation of the cell after several switching cycles is a crucial factor for further applications. Therefore, the IV characteristics were determined for several cycles of switching. Hereby, the cell was exposed to hydrogen atmosphere for 20 minutes at room temperature to reach the transparent state and tempered at $50{ }^{\circ} \mathrm{C}$ in ambient air for 20 minutes to return to the absorbing state. The long switching times were chosen, to guarantee complete switching of the cell. Figure 5 (c) presents the characteristic values of one cell for seven cycles of switching. The difference between "on" and "off" state can be clearly seen for all parameters. This shows that the switching effect is present even after several cycles. The change of $\mathrm{J}_{\mathrm{sc}}$ between "on" and "off" state can be directly attributed to the change of light absorbed by the device. The change of $\mathrm{V}_{\mathrm{oc}}$ is partially caused by the change of light confined to the cell, but also most probably a result of different band alignment between hydrogenated $\mathrm{Mg}$ and metallic $\mathrm{Mg}$ and the cell stack. The decreased FF in the hydrogenated state has been studied above and can be found for all cycles of switching. All three parameters influence the efficiency of the device which varies from 1.1 to 0.9 in the "on" state and from 0.6 to 0.7 in the "off" state. It is striking to see that the efficiency, $\mathrm{FF}, \mathrm{V}_{\mathrm{oc}}$ and $\mathrm{J}_{\mathrm{sc}}$ increase slightly during the first five cycles of switching. This is a result of the decrease of shunt conductivity from $\mathrm{G}_{\text {shunt }}=2.4 \mathrm{mS}$ after the first switching cycle to $G_{\text {shunt }}=2.1 \mathrm{mS}$ in the fifth cycle of switching. The shunt conductivity was also extracted from the diode model of the curves. A possible explanation might be that the hydrogen inside the device does not only affect the $\mathrm{Mg}$ layer, but also leads to an additional hydrogen passivation of the $\mathrm{Si}$ and Ge layers in combination with an annealing effect of the tempering at $50^{\circ} \mathrm{C}$. Further evidence for this theory might be that the improvement of the cell stops after five cycles, when hydrogen passivation and annealing effects reached their maximum impact. After five cycles of switching, the efficiency and FF drop to values of $\eta=0.9 \%$ and $\mathrm{FF}=43 \%$ in the "on" 
state. This can be mainly attributed to the decrease of $\mathrm{J}_{\mathrm{sc}}$, caused by the degradation of the switchable rear contact. Further switching reduces the cell parameters slightly. This leads to the assumption that the switching of the rear contact and the included mechanical stress on the interface does not change the structure of the $\mathrm{Si}$ and Ge layers in a detrimental way. Even after seven switching cycles an efficiency of $0.8 \%$ is reached. The decrease of efficiency might continue until a constant value of efficiency is reached, when the rear contact lost its switching capacity. The fact that the cells are not shunted after the switching process, suggests that the mechanical stress on the absorber layers upon hydrogenation is reduced. A volume increase of up to $30 \%$ of the $\mathrm{Mg}$ layer would have a disruptive effect on the ultra-thin p-i-n structure.

The degradation of the $\mathrm{Mg} / \mathrm{Ti} / \mathrm{Pd}$ rear contact can be studied in the light transmission of the device after several cycles of switching. The transmission of the device during the first switching cycles is shown in Figure 5 (d). While the "off" state only shows a small decrease of transparency, the light transmission is increased in the "on" state after five cycles of hydrogen absorption and desorption. The change of optical parameters is still significant, but reduced compared to the initial state. This leads to a reduction of light confinement in the absorber layers and therefore to a reduced photo current and efficiency. The fact that the reduction of transmittance in the transparent state is smaller than the increase in the light absorbing state, leads to the assumption, that the capability of loading with hydrogen is much less affected from repeated switching than the unloading of hydrogen. This implies, that by improving the desorption process of hydrogen from the rear contact, the degradation of the device could be further reduced. We found that after the fifth switching cycle, the difference in transparency between "on" and "off" state decreases linearly and tends to reach zero after ten cycles of switching. This leads to a decrease in absorption and therefore to a reduction of the PV performance and to a constant solar cell efficiency, independent of the solar cell state. A detailed analysis of the transmission data can be found in the supporting information. The highest value of transmission is reached in the "off" state at a wavelength of $830 \mathrm{~nm}$ with $T=21 \%$. In the visible wavelength the highest value is $T=17 \%$ at a wavelength of $625 \mathrm{~nm}$. In the cavity "on" state the transmission stays below $2.6 \%$ over the complete visible wavelength range. This means that the transmittance can be increased by a factor of eight after hydrogen absorption. Figure 5 (e) presents a chromaticity plot with CIE1931 spectrum for a standard observer at almost perpendicular angle of observation. The point presents the color impression of the cell in transparent state. The coordinates are $\mathrm{x}=0.39$ and $\mathrm{y}=0.39$. In the "on" state, the color changes only slightly to $\mathrm{x}=0.40$ and $\mathrm{y}=0.40$. This demonstrates color neutrality, which is important for window applications. The remaining slightly brownish color stems from the Ge and Si layers of the cell stack.

The implementation of the here presented switchable solar cell in a switchable window requires further improvements, which must be subject to further studies. First of all, the efficiency of the solar cell must be improved. This may be reached by improved electrical band-alignment and the development of 2D absorber materials. Secondly, the switching process must be adapted for larger scale applications. Research groups have already proposed strategies to implement gasochromic switchable layers into double glazing windows for improved energy efficiency of building facades and studied the temperature dependence of the switching process. ${ }^{5}$ This could serve as roadmap for the realization of a switchable solar cell. Further improvements could be reached by replacing the gasochromic rear contact with an electrochromic layer stack. ${ }^{22}$ It has to be kept in mind, that the energy cost to use a switchable solar window must not exceed its gain in electric power. The presented work tries to be the first step into this direction.

\section{Conclusion}

We showed that the combination of an ultra-thin a$\mathrm{Ge}: \mathrm{H}$ absorber layer and a switchable rear contact is able to reach switchable photocurrent generation, based on switchable absorption enhancement. This new approach decouples the development of the absorber layer from the optical switching. Our results show, that $\mathrm{Mg} / \mathrm{Pd}$ layer systems are a promising approach to reach color neutral switchable broadband absorption. Despite using a non-optimized device we reached an overall cell efficiency of $1.78 \%$. Guided 
by optical simulations it was shown, that light can be confined in an ultra-thin a-Ge:H layer for photocurrent generation and that the confinement can be reduced by the transition of $\mathrm{Mg}$ to $\mathrm{MgH}_{2}$. A quantum efficiency of $40 \%$ was reached in simulation and experimentally validated. The deleterious effect of mechanical stress induced to the layers during the absorption of hydrogen in the $\mathrm{Mg}$ layer was reduced by using a thin Ti interface. The negative effect of $\mathrm{Mg}$ on the $\mathrm{p}$-side of the $\mathrm{p}-\mathrm{i}-\mathrm{n}$ stack may be avoided, when the structure is transposed. This would result in $\mathrm{Mg}$ being in contact with the n-side of the cell, which may be beneficial for the cell performance due to the low work function of $\mathrm{Mg}$. This will be done in a future work, since the growth of a-Ge strongly depends on the substrate and requires further investigation. In conclusion, this study strengthens the idea that switchable cavities are a promising path to switchable photovoltaic windows. This research will serve as a base for future studies, where the durability of the layers is increased and alternative absorber layers are studied.

\section{Methods}

\section{Modeling}

The software package Scout/CODE by W. Theiss was used to create models for the transmission, the reflection and the absorption of the complete device by $1 \mathrm{D}$ transfer matrix method. Dielectric functions of the respective layers were created by fitting optical model functions to measured transmission and reflection data of single layers for near normal incidence. The refractive index data is shown in the supporting information. Refractive index data for $\mathrm{Ag}$ was implemented in SCOUT/CODE and obtained from Palik et $\mathrm{al}^{51}$. Refractive index data for $\mathrm{Pd}$ was used from the optical constant data base implemented in Scout/CODE. The IQE was simulated by using the local absorption spectra and assuming internal conversion efficiency from photons to charge carriers of one. Single diode curves were fitted to the measured IV data with the software IVFIT.

\section{Deposition and Characterization}

Silicon and germanium layers were deposited using plasma enhanced chemical vapor deposition (PECVD) in a Phoebus Lab Coater from Leybold Optics under a pressure of 1.5 to 4 mbar and a temperature of $200{ }^{\circ} \mathrm{C}$. $\mathrm{SiH}_{4}$ and $\mathrm{H}_{2}$ gas was used to create a-Si:H and $\mu \mathrm{c}-\mathrm{Si}: \mathrm{H}$ layers. The a-Si layers were deposited using $70 \mathrm{~W}$ power, while the $\mu \mathrm{c}-\mathrm{Si}: \mathrm{H}$ layers were created at $450 \mathrm{~W}$ power. The doped layers were deposited with the addition of $\mathrm{B}_{2} \mathrm{H}_{6}$ and $\mathrm{PH}_{3}$, respectively. For the deposition of a-Ge:H layers $\mathrm{GeH}_{4}$ and $\mathrm{H}_{2}$ gas was used for deposition at a power of $700 \mathrm{~W} . \mathrm{MoO}_{\mathrm{x}}, \mathrm{Ti}, \mathrm{Mg}$ and $\mathrm{Pd}$ layers were deposited by electron beam evaporation in a VTD box coater at a pressure of $10^{-6}$ mbar. The rate was controlled with a Piezo crystal. The layers were deposited on commercially available AZO from Solayer on $3 \mathrm{~mm}$ float glass. Thicknesses were measured with a Dektak 150 profilometer from Veeco and controlled by fitting of the optical models. Discrepancies between measurement and fitting of up to $2 \mathrm{~nm}$ were accepted. To establish the front contact, material was removed by laser scribing with a microSTRUCTvario Laser from 3DMicromac. The rear contact material has been deposited through masks in an area of $1 \times 1 \mathrm{~cm}^{2}$. To improve the electrical contact between the probe heads and the thin film (24 nm Mg $+6 \mathrm{~nm} \mathrm{Pd})$ back reflectors, a $300 \mathrm{~nm}$ thick $\mathrm{Mg}$ layer with width of $3 \mathrm{~mm}$ has been deposited on the side of the rear mirror. It is deposited in such way next to the switchable mirror that it does not influence the absorption of the layer stack and is not in any contact with the sample light beam. The front contact is also equipped with $300 \mathrm{~nm} \mathrm{Mg}$ for better electrical contact. Images of the sample are available in the supporting information. Optical measurements (transmission, reflection) were performed with a Cary UV-Vis 5000 with an integrating sphere. Scanning electron microscopy imaging and focused ion beam milling was done using a Zeiss Neon Crossbeam. The quantum efficiency was measured using a RR-2100 measurement system from LOT Oriel that uses LockIn detection of chopped light and a pre-amplifier for high current accuracy. IV curves were studied with a Solar Simulator WXS-155S-L2, AM1.5GMM from WACOM. Switching was done in a custom made setup using Arcal F5 gas from Air Liquide, with 5\%\% $\mathrm{H}_{2}$ in $\mathrm{N}_{2}$. The hydrogenated state was reached at room temperature after 15 minutes of constant gas flow. The dehydrogenation was done at $90{ }^{\circ} \mathrm{C}$ with ambient air for 15 minutes in an oven.

\section{Acknowledgements}


The authors thank Colleen Lattyak and Udayan Banik for proof-reading an early version of the manuscript and for helpful discussions. The authors also thank Nils Neugebohrn, Hosni Meddeb, Dennis Berends and Oleg Sergeev for helpful discussions.

\section{Associated Content}

Supporting Information Available: Graphs of modeled refractive index data for the deposited materials and photographs of the deposited cell structure; measured IV curves during first seven cycles of switching; analysis of degradation in transmittance; This material is available free of charge via the Internet at http://pubs.acs.org

\section{Funding Statement}

This work was funded by Deutsches Zentrum für Luft- und Raumfahrt e.V. (DLR). 
References

1. Baetens, R.; Jelle, B. P.; Gustavsen, A., Properties, requirements and possibilities of smart windows for dynamic daylight and solar energy control in buildings: A state-of-the-art review. Sol Energ Mat Sol C 2010, 94, 87-105. 2. Ke, Y.; Chen, J.; Lin, G.; Wang, S.; Zhou, Y.; Yin, J.; Lee, P. S.; Long, Y., Smart Windows: Electro-, Thermo-, Mechano-, Photochromics, and Beyond. Adv Energy Mater 2019, 9 (39), 1902066.

3. Wang, Y.; Runnerstrom, E. L.; Milliron, D. J., Switchable Materials for Smart Windows. Annual review of chemical and biomolecular engineering 2016, 7, 283-304.

4. Wittwer, V.; Datz, M.; Ell, J.; Georg, A.; Graf, W.; Walze, G., Gasochromic windows. Sol Energ Mat Sol C 2004, 84, 305-314.

5. Yoshimura, K.; Yamada, Y.; Bao, S.;

Tajima, K.; Okada, M., Preparation and characterization of gasochromic switchablemirror window with practical size. Sol Energ Mat Sol C 2009, 93, 2138-2142.

6. Granqvist, C. G., Recent progress in thermochromics and electrochromics: A brief survey. Thin Solid Films 2016, 614, 90-96. 7. Granqvist, C. G., Electrochromics for smart windows: Oxide-based thin films and devices. Thin Solid Films 2014, 564, 1-38. 8. Islam, S. M.; Hernandez, T. S.; McGehee, M. D.; Barile, C., Hybrid dynamic windows using reversible metal electrodeposition and ion insertion. Nat Energy 2019, 4 (3), 223-229.

9. $\quad$ Li, X. H.; Liu, C.; Feng, S. P.; Fang, N. X., Broadband Light Management with Thermochromic Hydrogel Microparticles for Smart Windows. Joule 2019, 3, 290-302.

10. Khandelwal, H.; Schenning, A. P. H. J.;

Debije, M. G., Infrared Regulating Smart Window Based on Organic Materials. Adv Energy Mater 2017, 7 (14).

11. Cao, D.; Xu, C.; Lu, W. Y.; Qin, C. X.;

Cheng, S., Sunlight-Driven Photo-

Thermochromic Smart Windows. Solar Rrl 2018, 2 (4), 1870163.

12. Lemarchand, P.; Doran, J.; Norton, B., Smart switchable technologies for glazing and photovoltaic applications. Energy Procedia 2014, 57, 1878-1887.

13. Biyik, E.; Araz, M.; Hepbasli, A.; Shahrestani, M.; Yao, R.; Shao, L.; Essah, E.; Oliveira, A. C.; del Caño, T.; Rico, E.; Lechón, J. L.; Andrade, L.; Mendes, A.; Atlı, Y. B., A key review of building integrated photovoltaic (BIPV) systems. Engineering Science and Technology, an International Journal 2017, 20 (3), 833-858. 14. Traverse, C. J.; Pandey, R.; Barr, M. C.; Lunt, R. R., Emergence of highly transparent photovoltaics for distributed applications. Nat Energy 2017, 2, 849-860.

15. Kwon, H. K.; Lee, K. T.; Hur, K.; Moon, S. H.; Quasim, M. M.; Wilkinson, T. D.; Han, J. Y.; Ko, H.; Han, I. K.; Park, B.; Min, B. K.; Ju, B. K.; Morris, S. M.; Friend, R. H.; Ko, D. H., Optically Switchable Smart Windows with Integrated Photovoltaic Devices. Adv Energy Mater 2015, 5 (3), 1401347.

16. Wang, J.; Zhang, L.; Yu, L.; Jiao, Z.; Xie, H.; Lou, X. W.; Sun, X. W., A bi-functional device for self-powered electrochromic window and self-rechargeable transparent battery applications. Nature communications 2014, 5, 4921.

17. Bechinger, C.; Ferrer, S.; Zaban, A.; Sprague, J.; Gregg, B. A., Photoelectrochromic windows and displays. Nature 1996, 383 (6601), 608-610.

18. Lampert, C. M., Large-area smart glass and integrated photovoltaics. Sol Energ Mat Sol C 2003, 76 (4), 489-499.

19. Murray, J.; Ma, D. K.; Munday, J. N., Electrically Controllable Light Trapping for SelfPowered Switchable Solar Windows. Acs Photonics 2017, 4 (1), 1-7.

20. Wheeler, L. M.; Moore, D. T.; Ihly, R.; Stanton, N. J.; Miller, E. M.; Tenent, R. C.; Blackburn, J. L.; Neale, N. R., Switchable photovoltaic windows enabled by reversible photothermal complex dissociation from methylammonium lead iodide. Nature communications 2017, 8 (1), 1722.

21. Lin, J.; Lai, M.; Dou, L.; Kley, C. S.; Chen, H.; Peng, F.; Sun, J.; Lu, D.; Hawks, S. A.; Xie, C.; Cui, F.; Alivisatos, A. P.; Limmer, D. T.; Yang, P., Thermochromic halide perovskite solar cells. Nature materials 2018, 17 (3), 261-267. 
22. Tajima, K.; Yamada, Y.; Bao, S.; Okada, M.; Yoshimura, K., Near colorless all-solid-state switchable mirror based on magnesiumtitanium thin film. J Appl Phys 2008, 103 (1), 013512.

23. Kats, M. A.; Sharma, D.; Lin, J.; Genevet, P.; Blanchard, R.; Yang, Z.; Qazilbash, M. M.; Basov, D. N.; Ramanathan, S.; Capasso, F., Ultrathin perfect absorber employing a tunable phase change material. Appl Phys Lett 2012, 101.

24. Chen, Y.; Duan, X.; Matuschek, M.; Zhou, Y.; Neubrech, F.; Duan, H.; Liu, N., Dynamic Color Displays Using Stepwise Cavity Resonators. Nano letters 2017, 17, 5555-5560. 25. Li, J.; Kamin, S.; Zheng, G.; Neubrech, F.; Zhang, S.; Liu, N., Addressable metasurfaces for dynamic holography and optical information encryption. Science Advances 2018, 4, 1-8. 26. Sterl, F.; Strohfeldt, N.; Walter, R.; Griessen, R.; Tittl, A.; Giessen, H., Magnesium as novel material for active plasmonics in the visible wavelength range. Nano letters 2015, 15, 7949-7955.

27. Palm, K. J.; Murray, J. B.; Narayan, T. C.; Munday, J. N., Dynamic Optical Properties of Metal Hydrides. Acs Photonics 2018, 5 (11), 4677-4686.

28. Isidorsson, J.; Giebels, I. A. M. E.; Arwin, H.; Griessen, R., Optical properties of MgH2 measured in situ in a novel gas cell for ellipsometry/spectrophotometry. 2003, 1-13.

29. Giebels, I. A. M. E.; Isidorsson, J.; Griessen, R., Highly absorbing black Mg and rare-earth-Mg switchable mirrors. Physical Review B 2004, 69, 205111.

30. Tajima, K.; Yamada, Y.; Bao, S.; Okada, M.; Yoshimura, K., Flexible all-solid-state switchable mirror on plastic sheet. Appl Phys Lett 2008, 92, 1-4.

31. Steenhoff, V.; Theuring, M.; Vehse, M.; von Maydell, K.; Agert, C., Ultrathin ResonantCavity-Enhanced Solar Cells with Amorphous Germanium Absorbers. Advanced Optical Materials 2015, 3 (2), 182-186.

32. Lattyak, C.; Steenhoff, V.; Gehrke, K.; Vehse, M.; Agert, C., Two-Dimensional Absorbers for Solar Windows: A Simulation.
Zeitschrift für Naturforschung A 2019, 74 (8), 683-688.

33. Steenhoff, V.; Osterthun, N.; Gehrke, K.; Vehse, M.; Agert, C. In Nanoabsorbers for PV New Potential Applications, 35th European Photovoltaic Solar Energy Conference and Exhibition, Brussels, Brussels, 2018; pp 51 - 54.

34. Farangis, B.; Nachimuthu, P.; Richardson, T. J.; Slack, J. L.; Meyer, B. K.; Perera, R. C. C.; Rubin, M. D., Structural and electronic properties of magnesium-3D transition metal switchable mirrors. Solid State Ionics 2003, 165, 309-314.

35. Richardson, T. J.; Slack, J. L.; Armitage, R. D.; Kostecki, R.; Farangis, B.; Rubin, M. D., Switchable mirrors based on nickel-magnesium films. Appl Phys Lett 2001, 78, 3047-3049.

36. Michaelson, H. B., The work function of the elements and its periodicity. J Appl Phys 1977, 48 (11), 4729-4733.

37. Yu, C.; Xu, S. Z.; Yao, J. X.; Han, S. W., Recent Advances in and New Perspectives on Crystalline Silicon Solar Cells with CarrierSelective Passivation Contacts. Crystals 2018, 8 (11), 430.

38. Jung, H.; Yuh, J.; Cho, S.; Lee, W., Effects of Ti interlayers on microstructures and hydrogen storage capacity in $\mathrm{Mg} / \mathrm{Pd}$ multilayer thin films. J Alloy Compd 2014, 601, 63-66.

39. Kats, M. A.; Blanchard, R.; Genevet, P.; Capasso, F., Nanometre optical coatings based on strong interference effects in highly absorbing media. Nature materials 2013, 12 (1), 20-4.

40. Krayer, L. J.; Kim, J.; Munday, J. N., Nearperfect absorption throughout the visible using ultra-thin metal films on index-near-zero substrates [Invited]. Optical Materials Express 2018, 9 (1), 330.

41. Krayer, L. J.; Kim, J.; Garrett, J. L.; Munday, J. N., Optoelectronic Devices on Indexnear-Zero Substrates. ACS Photonics 2019, 6 (9), 2238-2244.

42. Steenhoff, V.; Juilfs, M.; Ravekes, R. E.; Ahrlich, M.; Kellermann, M.; Siepmann, O.; Vehse, M.; Agert, C., Optimized Optical Field Profile in Resonant-Cavity-Enhanced a-Ge:H Nanoabsorber Solar Cells for Tandem Cell 
Application. IEEE Journal of Photovoltaics 2017, 7, 3-10.

43. Lee, K.-T.; Seo, S.; Lee, J. Y.; Guo, L. J., Strong Resonance Effect in a Lossy MediumBased Optical Cavity for Angle Robust Spectrum Filters. Advanced materials 2014, 26, 63246328.

44. Lattyak, C.; Ravekes, R. E.; Steenhoff, V.; Vehse, M.; Agert, C., Ultrathin Resonant-CavityEnhanced Amorphous Germanium Solar Cells on ZnO Honeycomb Electrodes. leee Journal of Photovoltaics 2018, 8 (1), 3-7.

45. Uchida, H. T.; Wagner, S.; Hamm, M.; Kürschner, J.; Kirchheim, R.; Hjörvarsson, B.; Pundt, A., Absorption kinetics and hydride formation in magnesium films: Effect of driving force revisited. Acta Materialia 2015, 85, 279289.

46. Mooij, L.; Dam, B., Hysteresis and the role of nucleation and growth in the hydrogenation of Mg nanolayers. Physical Chemistry Chemical Physics 2013, 15, 27822792.
47. Duan, X.; Griessen, R.; Wijngaarden, R. J.; Kamin, S.; Liu, N., Self-recording and manipulation of fast long-range hydrogen diffusion in quasifree magnesium. Phys. Rev. Materials 2018, 2, 85802.

48. Tong, H.; Yang, Z.; Wang, X.; Liu, Z.;

Chen, Z.; Ke, X.; Sui, M.; Tang, J.; Yu, T.; Ge, Z.;

Zeng, Y.; Gao, P.; Ye, J., Dual Functional Electron-Selective Contacts Based on Silicon Oxide/Magnesium: Tailoring Heterointerface Band Structures while Maintaining Surface Passivation. Adv Energy Mater 2018, 8 (16), 1702921.

49. Baldi, A.; Palmisano, V.; GonzalezSilveira, M.; Pivak, Y.; Slaman, M.; Schreuders, H.; Dam, B.; Griessen, R., Quasifree Mg-H thin films. Appl Phys Lett 2009, 95, 1-4.

50. Pundt, A., Hydrogen in Nano-sized Metals. Advanced Engineering Materials 2004, 6, 11-21.

51. Edward, D. Palik, Handbook of optical constants of solids. Academic, Orlando, Florida: 1985. 